\title{
BMJ Open Adaptive randomised controlled non- inferiority multicentre trial (the Ketodex Trial) on intranasal dexmedetomidine plus ketamine for procedural sedation in children: study protocol
}

\begin{abstract}
Naveen Poonai (10 , ${ }^{1,2}$ Kamary Coriolano, ${ }^{1}$ Terry Klassen, ${ }^{3,4}$ Anna Heath, ${ }^{5,6,7}$ Maryna Yaskina, ${ }^{8}$ Darcy Beer, ${ }^{9}$ Scott Sawyer, ${ }^{9}$ Maala Bhatt, ${ }^{10}$ April Kam, ${ }^{11}$ Quynh Doan, ${ }^{12}$ Vikram Sabhaney, ${ }^{12}$ Martin Offringa, ${ }^{13}$ Petros Pechlivanoglou, ${ }^{5}$ Serena Hickes, ${ }^{4}$ Samina Ali (iD , ${ }^{8,14}$ On behalf of KidsCAN PERC iPCT-SPOR (Innovative Paediatric Clinical Trials - Strategy for Patient Oriented Research) Ketodex Study Team
\end{abstract}

To cite: Poonai N, Coriolano K, Klassen T, et al. Adaptive randomised controlled non-inferiority multicentre trial (the Ketodex Trial) on intranasal dexmedetomidine plus ketamine for procedural sedation in children: study protocol. BMJ Open 2020;10:e041319. doi:10.1136/ bmjopen-2020-041319

- Prepublication history and additional material for this paper are available online. To view these files, please visit the journal online (http://dx.doi. org/10.1136/bmjopen-2020041319).

Received 07 June 2020 Revised 22 November 2020 Accepted 24 November 2020

Check for updates

(C) Author(s) (or their employer(s)) 2020. Re-use permitted under CC BY-NC. No commercial re-use. See rights and permissions. Published by BMJ.

For numbered affiliations see end of article.

Correspondence to

Dr Naveen Poonai;

naveen.poonai@|hsc.on.ca

\section{ABSTRACT}

Introduction Up to $40 \%$ of orthopaedic injuries in children require a closed reduction, almost always necessitating procedural sedation. Intravenous ketamine is the most commonly used sedative agent. However, intravenous insertion is painful and can be technically difficult in children. We hypothesise that a combination of intranasal dexmedetomidine plus intranasal ketamine (Ketodex) will be non-inferior to intravenous ketamine for effective sedation in children undergoing a closed reduction. Methods and analysis This is a six-centre, four-arm, adaptive, randomised, blinded, controlled, non-inferiority trial. We will include children 4-17 years with a simple upper limb fracture or dislocation that requires sedation for a closed reduction. Participants will be randomised to receive either intranasal Ketodex (one of three dexmedetomidine and ketamine combinations) or intravenous ketamine. The primary outcome is adequate sedation as measured using the Paediatric Sedation State Scale. Secondary outcomes include length of stay, time to wakening and adverse effects. The results of both per protocol and intention-to-treat analyses will be reported for the primary outcome. All inferential analyses will be undertaken using a response-adaptive Bayesian design. Logistic regression will be used to model the doseresponse relationship for the combinations of intranasal Ketodex. Using the Average Length Criterion for Bayesian sample size estimation, a survey-informed non-inferiority margin of $17.8 \%$ and priors from historical data, a sample size of 410 participants will be required. Simulations estimate a type II error rate of 0.08 and a type I error rate of 0.047 .

Ethics and dissemination Ethics approval was obtained from Clinical Trials Ontario for London Health Sciences Centre and McMaster Research Ethics Board. Other sites have yet to receive approval from their institutions. Informed consent will be obtained from guardians of all participants in addition to assent from participants. Study data will be submitted for publication regardless of results.
Strengths and limitations of this study

This study employs a response adaptive trial design to overcome gaps in our current understanding of the most effective dosing combination of intranasal Ketodex.

- To optimise trial efficiency, frequent assessment of the data will be used to adapt the trial to increase the number of participants receiving the more effective combination of Ketodex.

- This study involved a patient engagement strategy whereby patient partners informed the eligibility criteria and outcomes, and reviewed letters of information, consent and assent.

- We expect that current translation to practice may be limited by the high volume of intranasal drug required for older children unless a higher concentration of ketamine $(100 \mathrm{mg} / \mathrm{kg})$ becomes widely available.

Research nurses may become aware of group assignment and blinding will only be possible for outcome assessors.

Trial registration number NCT0419525.

\section{INTRODUCTION}

Orthopaedic injuries comprise more than $10 \%$ of emergency department (ED) visits in children, ${ }^{12}$ and $25 \%-50 \%$ of children will sustain a fracture before the age of 16 years. ${ }^{3}$ Between $20 \%$ and $40 \%$ of extremity fractures in children require a closed reduction, ${ }^{45}$ often necessitating procedural sedation. The demand for procedural sedation in children outside the operating room is increasing at a rate of $10 \%$ annually. ${ }^{67}$ As such, the 
placement of an intravenous catheter for procedural sedation is extremely common. However, children rate intravenous insertion as one of the most painful hospital experiences, second only to the painful condition itself. ${ }^{8}$ intravenous insertion can be more technically difficult in children because of smaller veins ${ }^{9}$ and lack of cooperation, ${ }^{89}$ often leading to multiple intravenous attempts.

Intranasal medications may obviate the need for distressing intravenous placement and offer a technically easier and pain-free approach to procedural sedation. ${ }^{10}$ This may have widespread applicability in patients with difficult intravenous access, resource-limited settings, needle phobia or when experience placing an intravenous is limited. Ketamine is the most commonly used sedative agent for fracture reduction in children ${ }^{11}$ and intranasal ketamine has been found to be effective for fracture pain, ${ }^{10}$ some procedural pain, ${ }^{12}$ anaesthetic preinduction $^{13}$ and diagnostic imaging. ${ }^{14}$ However, neither agent has been studied for procedural sedation to reduce fractures or dislocations. Dexmedetomidine is a central alpha 2-adrenergic receptor agonist with analgesic and anxiolytic properties and is effective for procedural anxiety in children in its intravenous form. ${ }^{215} 16$ A recent systematic review of intranasal dexmedetomidine in children undergoing painful procedures (dental procedures, venipuncture and laceration repair) found doses ranging from 1 to $4 \mathrm{mcg} / \mathrm{kg}$ were well tolerated and superior to conventional sedatives (oral chloral hydrate and oral and intranasal midazolam) in providing adequate sedation. ${ }^{17}$

However, procedural sedation for orthopaedic reduction may require agents with greater sedative and analgesic potency. Preliminary evidence suggests that a combination of dexmedetomidine and ketamine may be superior than either agent alone. ${ }^{18} 19$ A combination of intranasal ketamine and intranasal dexmedetomidine ('Ketodex') theoretically combines the analgesic efficacy of ketamine ${ }^{20}$ with the sedative efficacy of dexmedetomidine. ${ }^{21}$ However, there remains uncertainty regarding the ideal combination of these two agents. In order to provide robust evidence supporting an alternate approach that precludes the need for an intravenous in children undergoing sedation, a response-adaptive Bayesian design will be used to determine the most effective combination of Ketodex and to test our hypothesis of whether this combination is non-inferior to intravenous ketamine.

\section{METHODS AND ANALYSIS \\ Design}

This is a six-centre, four-arm, randomised, blinded, double-dummy, controlled, parallel group, adaptive dosefinding, non-inferiority, phase II/III trial. The trial will determine whether intranasal Ketodex is non-inferior to intravenous ketamine for children undergoing procedural sedation and analgesia determine the optimal dosing combination for intranasal Ketodex. The study protocol is reported using the SPIRIT-PRO reporting guidelines. $^{22}$

\section{Study setting}

This study will be conducted in six paediatric EDs across Canada: (1) Children's Hospital at London Health Sciences Centre (London, Ontario) (coordinating site); (2) Stollery Children's Hospital (Edmonton, Alberta); (3) BC Children's Hospital (Vancouver, British Columbia); (4) Winnipeg Children's Hospital (Winnipeg, Manitoba); (5) CHEO (Ottawa, Ontario); and (6) McMaster Children's Hospital (Hamilton, Ontario). The annual ED census for recruiting centres ranges from 30000 to 70000 patient visits.

\section{Eligibility}

Children will be eligible if they meet all of the following criteria: (1) provision of signed and dated informed consent form; (2) stated willingness to comply with all study procedures and availability for the duration of the study; (3) deemed by treating physician to require procedural sedation; (4) aged 4-17 years; (5) weighing up to and including $60 \mathrm{~kg}$; (6) one of the following injuries: forearm fracture, metacarpal or phalangeal fracture, or dislocation of a shoulder or elbow; (7) closed reduction expected to take no more than $5 \mathrm{~min}$ to reduce (as determined by the procedure physician and not including cast or splint application); and (8) both nares are fully patent. Exclusion criteria are listed in table 1.

\section{Interventions and permissible cointerventions}

Eligible participants will be randomised to intranasal Ketodex or intravenous ketamine with a 3:2 allocation ratio. Participants receiving intranasal Ketodex will be further adaptively randomised to three alternative combinations where the randomisation ratio is proportional to the posterior probability that a combination is optimal in terms of providing adequate sedation. A double-dummy approach will be used to overcome the possibility of unmasking due to perceptible differences in interventional routes. This involves each participant receiving both an intranasal and intravenous intervention, only one of which is the real drug. Both intranasal and intravenous interventions will be administered through any of the following:

1. Dexmedetomidine (Pfizer, Kirkland, Quebec), single dose, $4 \mathrm{mcg} / \mathrm{kg}(0.04 \mathrm{~mL} / \mathrm{kg})$ of $100 \mathrm{mcg} / \mathrm{mL}$ solution, maximum of $200 \mathrm{mcg}(2 \mathrm{~mL})$, then ketamine (Sandoz, Mississauga, Ontario), single dose, $2 \mathrm{mg}$ / $\mathrm{kg}(0.04 \mathrm{~mL} / \mathrm{kg})$ of $50 \mathrm{mg} / \mathrm{mL}$ solution, maximum of $200 \mathrm{mg}(4 \mathrm{~mL})$ (D4K2), both delivered intranasally using a mucosal atomiser device (MAD) and divided to both nares and $0.9 \%$ normal saline $0.03 \mathrm{~mL} / \mathrm{kg}$ delivered intravenously to a maximum of $2 \mathrm{~mL}$.

2. Dexmedetomidine (Pfizer), single dose, $3 \mathrm{mcg} / \mathrm{kg}$ $(0.03 \mathrm{~mL} / \mathrm{kg})$ of $100 \mathrm{mcg} / \mathrm{mL}$ solution, maximum of $200 \mathrm{mcg}(2 \mathrm{~mL})$, then ketamine (Sandoz), single dose, $3 \mathrm{mg} / \mathrm{kg}(0.06 \mathrm{~mL} / \mathrm{kg})$ of $50 \mathrm{mg} / \mathrm{mL}$ solution, maximum of $300 \mathrm{mg}(6 \mathrm{~mL})$ (D3K3), both delivered intranasally using MAD and divided to both nares and $0.9 \%$ 
Table 1 Trial registration dataset

\section{Data category}

Primary registry and trial identifying number

Date of registration in primary registry

Secondary identifying numbers

Sources of monetary or material support

\section{Information}

clinicaltrials.gov

15 August 2019

Clinical Trials Ontario \# 1987

Canadian Institutes of Health Research SPOR Innovative Clinical Trials Grant (MYG-151207)

Physicians Services Incorporated Foundation

Academic Medical Organisation of Southwestern Ontario

Ontario Ministry of Economic Development, Job Creation and Trade Early Researcher Award

Primary sponsor
Secondary sponsor
Contact for public queries
Contact for scientific queries
Public title

Scientific title Lawson Health Sciences Research Institute

$-$

Dr Naveen Poonai, naveen.poonai@Ihsc.on.ca

Dr Naveen Poonai, naveen.poonai@Ihsc.on.ca

The Ketodex study

Adaptive randomised controlled non-inferiority multicentre trial (the Ketodex trial) on intranasal dexmedetomidine plus ketamine for procedural sedation in children study protocol

Countries of recruitment Canada

Health conditions or problems studied

Interventions
Fracture, dislocation

1. Dexmedetomidine (Pfizer, Kirkland, Quebec), single-dose, $4 \mathrm{mcg} / \mathrm{kg}$ $(0.04 \mathrm{~mL} / \mathrm{kg})$ of $100 \mathrm{mcg} / \mathrm{mL}$ solution, maximum of $200 \mathrm{mcg}(2 \mathrm{~mL})$, then ketamine (Sandoz, Mississauga, Ontario), single dose, $2 \mathrm{mg} /$ $\mathrm{kg}(0.04 \mathrm{~mL} / \mathrm{kg})$ of $50 \mathrm{mg} / \mathrm{mL}$ solution, maximum of $200 \mathrm{mg}(4 \mathrm{~mL})$ (D4K2), both delivered intranasally using a MAD and divided to both nares, and $0.9 \%$ normal saline $0.03 \mathrm{~mL} / \mathrm{kg}$ delivered intravenously to a maximum of $2 \mathrm{~mL}$ or

2. Dexmedetomidine (Pfizer), single dose, $3 \mathrm{mcg} / \mathrm{kg}(0.03 \mathrm{~mL} / \mathrm{kg})$ of $100 \mathrm{mcg} / \mathrm{mL}$ solution, maximum of $200 \mathrm{mcg}(2 \mathrm{~mL})$ then ketamine (Sandoz), single dose, $3 \mathrm{mg} / \mathrm{kg}(0.06 \mathrm{~mL} / \mathrm{kg})$ of $50 \mathrm{mg} / \mathrm{mL}$ solution, maximum of $300 \mathrm{mg}(6 \mathrm{~mL})(\mathrm{D} 3 \mathrm{~K} 3)$, both delivered intranasally using a MAD and divided to both nares and $0.9 \%$ normal saline $0.03 \mathrm{~mL} / \mathrm{kg}$ delivered intravenously to a maximum of $2 \mathrm{~mL}$ or

3. Dexmedetomidine (Pfizer), single dose, $2 \mathrm{mcg} / \mathrm{kg}(0.02 \mathrm{~mL} / \mathrm{kg})$ of $100 \mathrm{mcg} / \mathrm{mL}$ solution, maximum of $200 \mathrm{mcg}(2 \mathrm{~mL})$ then ketamine (Sandoz), single dose, $4 \mathrm{mg} / \mathrm{kg}(0.08 \mathrm{~mL} / \mathrm{kg})$ of $50 \mathrm{mg} / \mathrm{mL}$ solution, maximum of $400 \mathrm{mg}(8 \mathrm{~mL})$ (D2K4), both delivered intranasally using MAD and divided to both nares and $0.9 \%$ normal saline $0.03 \mathrm{~mL} / \mathrm{kg}$ delivered intravenously to a maximum of $2 \mathrm{~mL}$ or

4. Ketamine, single dose, $1.5 \mathrm{mg} / \mathrm{kg}(0.03 \mathrm{~mL} / \mathrm{kg})$ of $50 \mathrm{mg} / \mathrm{mL}$ solution delivered intravenously, to a maximum of $100 \mathrm{mg}(2 \mathrm{~mL})$ and two aliquots of $0.9 \%$ normal saline in three possible combinations: (1) $0.04 \mathrm{~mL} / \mathrm{kg}(\max 2 \mathrm{~mL})$ then $0.04 \mathrm{~mL} / \mathrm{kg}(\max 4 \mathrm{~mL})$ (placebo D4K2), (2) $0.03 \mathrm{~mL} / \mathrm{kg}(\max 2 \mathrm{~mL})$ then $0.06 \mathrm{~mL} / \mathrm{kg}(\max 6 \mathrm{~mL})$ (placebo D3K3), (3) $0.02 \mathrm{~mL} / \mathrm{kg}(\max 2 \mathrm{~mL})$ then $0.08 \mathrm{~mL} / \mathrm{kg}$ (max $8 \mathrm{~mL}$ ) (placebo D2K4), delivered intranasally using MAD and divided to both nares. 
Table 1 Continued

Data category

Key inclusion and exclusion criteria

\section{Information}

General criteria

1. Provision of signed and dated informed consent form.

2. Stated willingness to comply with all study procedures and availability for the duration of the study.

3. Deemed by treating physician to require procedural sedation. Inclusion criteria

1. Children presenting to the paediatric emergency departments of participating sites age 4-17 years.

2. Weighing up to and including $60 \mathrm{~kg}$.

3. One of the following injuries:

4. Forearm fracture.

5. Metacarpal or phalangeal fracture.

6. Dislocation of a shoulder or elbow.

7. Closed reduction expected to take no more than $5 \mathrm{~min}$ of manipulation to reduce (as determined by the procedure physician and not including cast or splint application).

Both nares are fully patent.

Exclusion criteria

Previous hypersensitivity reaction to ketamine or dexmedetomidine including rash, difficulty breathing, hypotension, apnea or laryngospasm.

Suspected globe rupture.

Concomitant traumatic brain injury with known intracranial haemorrhage.

Uncontrolled hypertension.

Nasal bone deformity or septal deviation.

Poor English or French fluency in the absence of native language interpreter.

American Society of Anesthesiologists Class III or greater.

Previous diagnosis of schizophrenia or active psychosis as per the treating physician.

Neurocognitive impairment that precludes the ability to self-report pain and satisfaction.

More than one fracture or dislocation requiring reduction.

Haemodynamic compromise as per the treating physician.

Glasgow coma score $<15$.

Previous sedation with ketamine within 24 hours.

Fracture is comminuted or associated with a dislocation.

Participant has undergone a haematoma block within 24 hours.

Previous enrolment in the trial.

Suspected pregnancy.

Congenital heart disease or known cardiac dysrhythmia.

Known or suspected hepatic impairment.

Known renal insufficiency.

Uncorrected mineralocorticoid deficiency.

Obstructive sleep apnoea.

\section{Study type}

Randomised, blinded, double-dummy, controlled, parallel group, adaptive dose-finding, non-inferiority, phase II/III trial

Date of first enrolment

11 March 2020

$\begin{array}{ll}\text { Sample size } & 410 \\ \text { Recruitment status } & \text { Actively recruiting }\end{array}$


Table 1 Continued

\section{Data category}

Primary outcomes
Key secondary outcomes

\section{Information}

Adequate sedation for the duration of the procedure. For closed reduction, this is defined as the interval of time from the first application of traction or manipulation of the injured limb for the purpose of anatomical realignment to the last application of a realigning force. Adequate is defined as fulfilment of all three of the following criteria:

1. A PSSS score of 2 or 3 for the duration of the procedure.

2. No additional medication is given during the procedure for the purpose of sedation.

3. The patient did not actively resist, cry or require physical restraint for completion of the closed reduction.

Length of stay (min): defined as the time recorded in the medical record between triage and discharge. This is an important consideration to the uptake of intranasal Ketodex in practice.

Time to wakening: defined as the duration of time between the first pair or intranasal sprays to the first PSSS score of $>3$, postclosed reduction. AEs: AEs are based on Health Canada reporting standards. They include nasal irritation, which will be assessed using the Faces Pain Scale Revised immediately prior to discharge and maladaptive behaviours 24-48 hours postrecovery, which will be assessed using the PHBQ. The FPS-R will be administered using an iPad and the PHBQ will be administered through an automated email survey from REDCap or by telephone.

\begin{tabular}{|c|c|}
\hline Other endpoints & $\begin{array}{l}\text { 1. Length of stay due to PSA is the time interval between the first pair of } \\
\text { intranasal sprays to discharge. } \\
\text { 2. Duration of procedure is the time interval between the first pair } \\
\text { of intranasal sprays/intravenous dose to the end of cast or splint } \\
\text { application. } \\
\text { 3. Length of stay is the time interval between triage assessment and } \\
\text { discharge. } \\
\text { 4. Caregiver, participant, bedside nurse or respiratory therapist, and } \\
\text { physician satisfaction with sedation will be recorded at the index visit } \\
\text { using a Visual Analogue Scale. For the caregiver and participant, the } \\
\text { following questions will be posed immediately prior to discharge: how } \\
\text { satisfied were you with your child's sedation? (caregiver); how happy } \\
\text { were you with your sleep? (participant). For the healthcare providers, } \\
\text { the following question will be posed immediately following cast/splint } \\
\text { application: how satisfied were you with the level of sedation in your } \\
\text { patient? } \\
\text { 5. Nasal irritation: discomfort associated with nasal sprays (if recalled), } \\
\text { assessed by the research nurse using the FPS-R at discharge. } \\
\text { 6. Volume of intranasal intervention received compared with volume of } \\
\text { intranasal intervention calculated to be received will be recorded at the } \\
\text { index visit. } \\
\text { 7. Adjunctive intravenous therapy and medications (eg, analgesics, } \\
\text { antibiotics, antiemetics and fluids) will be recorded at the index visit. } \\
\text { 8. Presedation pain will be recorded by the research nurse from the } \\
\text { participant using the FPS-R immediately prior to the first pair of } \\
\text { intranasal sprays. } \\
\text { 9. Patient preference for the method of sedation will be recorded at the } \\
\text { index visit by asking the participant: if you were to be put to sleep } \\
\text { again for an injury, what would you prefer, an intravenous needle or } \\
\text { nasal sprays (choose one)? }\end{array}$ \\
\hline
\end{tabular}

Continued 
Table 1 Continued

AE, adverse event; FPS-R, faces pain scale-revised; MAD, mucosal atomiser device; PHBQ, posthospital behaviour questionnaire; PSA, Procedural sedation and analgesia; PSSS, paediatric sedation state scale; REDCap, Research Electronic Data Capture .

normal saline $0.03 \mathrm{~mL} / \mathrm{kg}$ delivered intravenously to a maximum of $2 \mathrm{~mL}$.

3. Dexmedetomidine (Pfizer), single dose, $2 \mathrm{mcg} / \mathrm{kg}$ $(0.02 \mathrm{~mL} / \mathrm{kg})$ of $100 \mathrm{mcg} / \mathrm{mL}$ solution, maximum of $200 \mathrm{mcg}(2 \mathrm{~mL})$, then ketamine (Sandoz), single dose, $4 \mathrm{mg} / \mathrm{kg}(0.08 \mathrm{~mL} / \mathrm{kg})$ of $50 \mathrm{mg} / \mathrm{mL}$ solution, maximum of $400 \mathrm{mg}(8 \mathrm{~mL})$ (D2K4), both delivered intranasally using MAD and divided to both nares and $0.9 \%$ normal saline $0.03 \mathrm{~mL} / \mathrm{kg}$ delivered intravenously to a maximum of $2 \mathrm{~mL}$.

4. Ketamine, single dose, $1.5 \mathrm{mg} / \mathrm{kg}(0.03 \mathrm{~mL} / \mathrm{kg})$ of $50 \mathrm{mg} / \mathrm{mL}$ solution delivered intravenously, to a maximum of $100 \mathrm{mg}(2 \mathrm{~mL})$ and two aliquots of $0.9 \%$ normal saline in three possible combinations: (1) $0.04 \mathrm{~mL} /$ $\mathrm{kg}(\max 2 \mathrm{~mL})$ then $0.04 \mathrm{~mL} / \mathrm{kg}(\max 4 \mathrm{~mL})$ (placebo $\mathrm{D} 4 \mathrm{~K} 2)$, (2) $0.03 \mathrm{~mL} / \mathrm{kg}(\max 2 \mathrm{~mL})$ then $0.06 \mathrm{~mL} /$ $\mathrm{kg}(\max 6 \mathrm{~mL})$ (placebo D3K3), and (3) $0.02 \mathrm{~mL} / \mathrm{kg}$ $(\max 2 \mathrm{~mL})$ then $0.08 \mathrm{~mL} / \mathrm{kg}(\max 8 \mathrm{~mL})$ (placebo D2K4), delivered intranasally using MAD and divided to both nares.

Participants will be positioned in a recumbent supine position at $45^{\circ}$. Each pair of sprays will be separated by at least $60 \mathrm{~s}$. The physician or their designate must administer the intravenous intervention $30-40 \mathrm{~min}$ after the intranasal dexmedetomidine/saline sprays. The research or bedside nurses will administer intranasal interventions, and the physician or their designate will administer intravenous interventions (figure 1).

All participants will receive continuous cardiorespiratory monitoring, consisting of five-lead continuous ECG, oxygen saturation and blood pressure, with consideration of capnography. This will commence immediately prior to administration of the intervention and will continue until the participant is awake.

The treating physician will not be permitted to prescribe any sedative, anxiolytic or analgesic cointervention within $15 \mathrm{~min}$ of the study intervention. Analgesics for pain and antiemetics may be given at any time at the discretion of the clinician or nurse outside the procedural sedation period. Anxiolytics for emergence agitation or anxiety may be given following the closed reduction. Any prescribed home or over-the-counter medications may be given at any time during the index visit with the exception of sedatives.
Rescue sedation may be provided if, after $1 \mathrm{~min}$ following the intravenous intervention, either of the following conditions are met: (1) the participant is still responding to surroundings or (2) procedure has begun and the participant's vocalisations are consistent with pain, or the participant is withdrawing or localising to pain.

\section{Randomisation and allocation concealment}

Randomisation will be performed in two stages as follows: 1. First, the pharmacy at each site will randomise participants in a 3:2 allocation ratio to intranasal Ketodex or intravenous ketamine and will adapt a previously published design. ${ }^{23}$ This stage will be stratified by site and will be randomised in a block size known only to the statistical team.

2. Second, participants in the Ketodex group will be adaptively randomised using REDCap to three possible combinations of intranasal ketamine and intranasal dexmedetomidine in a 1:1:1 ratio for the first 150 participants: $2 \mathrm{mg} / \mathrm{kg}$ intranasal ketamine $+4 \mu \mathrm{g} / \mathrm{kg}$ intranasal dexmedetomidine or $3 \mathrm{mg} / \mathrm{kg}$ intranasal ketamine $+3 \mu \mathrm{g} / \mathrm{kg}$ intranasal dexmedetomidine or $4 \mathrm{mg} /$ $\mathrm{kg}$ intranasal ketamine $+2 \mu \mathrm{g} / \mathrm{kg}$ intranasal dexmedetomidine). After the first 150 participants, the randomisation ratio will be adapted after every 50 recruited participants. The allocation ratio will be set equal to the posterior probability that a given dose is the most effective given all the available data at each interim analysis (200, 250, 300 and 350 participants). The number of participants randomised to each combination will be adapted throughout the trial to increase the number of participants receiving the more effective intranasal Ketodex combination. Intranasal Ketodex combinations will be dropped if the probability that this dose is optimal falls below 0.05 . Two combinations will be dropped if there is a greater than $95 \%$ chance that a single combination is optimal. If all dose combinations have a probability of being the most effective of less than $50 \%$ after 250 participants have been enrolled in the trial, the safety profile will be evaluated to determine the most promising combination in conjunction with the data safety monitoring board (DSMB). This

\begin{tabular}{|l|l|l|l|l|}
\hline \multicolumn{1}{l}{$\mathbf{0} \mathbf{~ m i n}$} & $\mathbf{m i n}$ & $\mathbf{2 5 - 4 0 ~} \mathbf{m i n}$ & $\mathbf{2 6} \mathbf{~ m i n}$ \\
\hline Pain score & $\begin{array}{l}\text { Intranasal } \\
\text { dexmedetomidine } \\
\text { or saline }\end{array}$ & $\begin{array}{l}\text { Intranasal } \\
\text { ketamine } \\
\text { or saline }\end{array}$ & $\begin{array}{l}\text { Intravenous } \\
\text { ketamine or } \\
\text { saline }\end{array}$ & Closed reduction \\
\hline
\end{tabular}

Figure 1 Intervention administration. 
will be used as the single intranasal Ketodex comparator for the remainder of the trial.

NB. Participants in the intravenous ketamine group will also undergo the second stage of randomisation but will receive saline in the identical volumes to the active drug, that is, two aliquots of $0.9 \%$ normal saline in three possible combinations: (1) $0.04 \mathrm{~mL} / \mathrm{kg}$ (max $2 \mathrm{~mL}$ ) then $0.04 \mathrm{~mL} / \mathrm{kg}(\max 4 \mathrm{~mL}),(2) 0.03 \mathrm{~mL} / \mathrm{kg}(\max 2 \mathrm{~mL})$ then $0.06 \mathrm{~mL} / \mathrm{kg}(\max 6 \mathrm{~mL})$ and $(3) 0.02 \mathrm{~mL} / \mathrm{kg}(\max$ $2 \mathrm{~mL})$ then $0.08 \mathrm{~mL} / \mathrm{kg}(\max 8 \mathrm{~mL})$.

The allocation tables will be generated by the data coordinating centre (DCC) statistician at the Women and Children's research Institute at the University of Alberta using $\mathrm{R}$. The allocation tables across the different dose combinations will be updated at 150, 200, 250, 300 and 350 participants. An independent statistician will validate the tables and the code that was used to create them. The tables will then be provided to site pharmacies for creation of the study kits, ensuring that study staff remain blinded. Site pharmacies will prepare identically appearing study kits containing the intravenous and intranasal interventions in accordance with the allocation tables.

\section{Blinding}

Blinded parties include the participant, caregiver, research nurse videotaping the procedure and outcome assessor. Participants should not be aware of group assignment because $0.9 \%$ normal saline, dexmedetomidine and ketamine solutions are odourless, colourless and tasteless. Kits containing the interventions will be identically appearing, differing only by a study number. However, differences in onset of sedation between intravenous ketamine and intranasal Ketodex risk unblinding the sedating physician, research nurse and outcome assessor. To minimise this risk, the intravenous intervention will be given $30-40 \mathrm{~min}$ following the intranasal dexmedetomidine/saline sprays due to intranasal dexmedetomidine's longer onset of action (20-30 min $)^{21}$ versus intravenous ketamine $(1 \mathrm{~min}) .{ }^{24}$ To increase the probability that both components of intranasal Ketodex are clinically effective at the same time, a green-labelled vial containing intranasal dexmedetomidine or saline will be administered first, followed by a white-labelled vial containing either intranasal ketamine or saline.

The research nurse will record the video of the participant's entire body (including the face) starting immediately prior to the closed reduction and continuing until the reduction is complete. Two trained and independent outcome assessors remote from the clinical encounter and unaware of the study objectives will score sedation using the Paediatric Sedation State Scale (PSSS) (and determine the primary outcome) every $30 \mathrm{~s}$ for the entire length of the video. The second outcome assessor will score a randomly selected $25 \%$ sample of the participant videos to generate an inter-rater agreement. Flags will alert the outcome assessor when the closed reduction procedure is started and completed.

\section{Unblinding procedures}

The DSMB may request unblinding directly from the DCC statistician. Urgent unblinding may be done if the participant suffers an adverse event (AE), the management of which is predicated on knowing the group assignment. The research nurse will log into a secure web-based unblinding system with REDCap, where the study medication will be revealed only to the treating physician. Thus, caregivers, children and research staff, including outcome assessors, will remain blinded.

\section{Recruitment}

Potential participants will be screened and enrolled consecutively during the hours of research nurse availability ( $\leq 8$ hours/day, 7 days/week). Families will provide verbal consent for eligibility screening by the research nurse. For patients who pass initial screening, the research nurse will confirm eligibility with the ED physician or their designate (any clinician who has the capacity to assess, manage and discharge a patient). If eligibility is confirmed, the research nurse will explain the study protocol and seek informed consent (and assent when appropriate) (see online supplemental files 1 and 2). Mature minor consent forms will be available for both accompanied and unaccompanied minors. Research nurse will record basic demographic features and eligibility criteria of all children with an orthopaedic injury requiring procedural sedation during their availability, whether randomised or not, to assess for enrolment bias.

\section{Data collection}

All outcomes and endpoints apart from the determination of adequate sedation (the primary outcome) will be collected by the research nurse and recorded using REDCap and a Wi-Fi-enabled iPad device.

The research nurse will record a video of the participant's closed reduction using a Canon VIXIA HF R700 camcorder mounted on a tripod. Once data collection is completed, the video file will be uploaded onto a shared drive, which will be accessed only by the two outcome assessors. Training for the outcome assessors will consist of a 1-hour PowerPoint presentation that outlined PSSS scoring and hands-on training using videos of 25 children who underwent procedural sedation.

Following the application of a cast or splint, the research nurse will obtain satisfaction scores from the sedating provider, bedside nurse and participant. Research nurses will be trained on the recognition and definition of all expected and unexpected AEs. ${ }^{24-26}$ The research nurse will record AEs from the medical record and queries from healthcare staff during sedation and recovery. Uncertainty will be clarified with the sedating physician. All AEs will be recorded except for expected physiological effects of ketamine such as elevated blood pressure and heart rate, increased oral secretions, nystagmus, enhanced skeletal muscle tone, flushing and confusion on wakening. ${ }^{27} 28$

When the participant is awake, the research nurse will ask participants to rate their nasal irritation related to 
the intranasal sprays using the FPS-R. To detect maladaptive behaviours following discharge, the caregiver will be surveyed either by telephone or automated email survey administered by REDCap 24-48 hours following discharge using the Posthospital Behaviour Questionnaire (PHBQ) ${ }^{29} \mathrm{~A}$ schedule of activities is shown in table 2.

\section{Outcome measures}

The primary efficacy outcome is adequate sedation for the duration of the procedure as we believed this to be the most salient patient-oriented outcome. Sedation will be deemed 'adequate' if all of the following conditions are met: (1) a PSSS score of 2 or 3 for the duration of closed reduction; (2) no additional medication is given during the procedure for the purpose of sedation; and (3) the patient did not actively resist, cry or require physical restraint for completion of the procedure. The PSSS (figure 2) is an instrument developed for assessment of sedation in children aged 1-7 years undergoing laceration repair using video scoring. ${ }^{30}$ The PSSS is scored from 0 to 5 , with a higher number indicating a lesser degree of sedation. The PSSS assesses pain as well as oversedation and undersedation. Adequate sedation is a score of 2 or $3^{30}$ and represents a level of sedation that correlates with a lack of pain, distress or oversedation or undersedation; optimal for procedural sedation as outlined by the Joint Commission, the American Academy of Paediatrics ${ }^{31}$ and the American Society of Anaesthesia. ${ }^{32}$

Secondary outcomes include the following:

1. Length of stay is the time interval between triage and discharge.

2. Time to wakening is the time interval between the first pair or intranasal sprays to the first PSSS score of $>3$ postclosed reduction as determined by the research nurse.

3. AEs are based on Health Canada reporting standards (see online supplemental file 3). They include nasal irritation, which will be assessed using the FPS- $\mathrm{R}^{33}$ immediately prior to discharge and maladaptive behaviours 24-48 hours postrecovery, which will be assessed using the PHBQ. ${ }^{29}$ The FPS-R will be administered using an iPad, and the PHBQ will be administered through an automated email survey from REDCap or by telephone. All AEs will be classified according to the Medical Dictionary for Regulatory Activities (MedDRA).

Other endpoints are listed in table 1.

\section{Sample size}

Two surveys were disseminated to over 200 paediatric and general emergency physicians across Canada. We presented the case of a 5-year-old girl meeting the study's inclusion criteria. We asked respondents to choose singledose intravenous or intranasal ketamine for procedural sedation and to specify the largest percentage of children who failed intranasal sedation they would be willing to accept in order to routinely attempt the intranasal route first. From these surveys, the average non-inferiority margin $\eta$ was $17.8 \%$. To determine a maximum sample size, we used the Average Length Criterion (ALC) for Bayesian sample size estimation. ${ }^{34}$ This method selects the smallest sample size required to ensure that the $95 \%$ posterior credible interval has an average length of 0.07 . We also considered four alternative randomisation strategies of intravenous ketamine to intranasal Ketodex: 1:4, 3:7, 2:3 and 1:1. The ALC determines the maximum sample size and the randomisation ratio between intravenous ketamine and intranasal Ketodex. First, we selected the smallest sample size for which the average length of the $95 \%$ posterior credible interval fell below 0.07 . For this sample size, we then selected the randomisation ratio that led to the most balanced trial, provided the average length of the $95 \%$ posterior credible interval remained below 0.07 . We used 2000 simulations to estimate the average posterior credible interval; the posteriors were approximated using 2000 simulation, for sample sizes increasing in increments of 10 , across all four randomisation regimes. Based on this analysis, the sample size for the Ketodex trial is 410 patients randomised at a 3:2 ratio of intranasal Ketodex to intravenous ketamine.

\section{Statistical methods}

The results of both per protocol and intention to treat analyses will be reported for the primary outcome, with the intention-to-treat analysis being taken as the primary analysis. Demographic data will be summarised using frequencies and percentages for categorical variables and means, medians, SD and IQRs for continuous variables. Length of stay, onset of sedation and duration of sedation will be analysed using a linear dose-response model to estimate the mean duration for the optimal intranasal Ketodex combination. Adverse effects (AEs) will be analysed using a logistic dose-response model for intranasal Ketodex and a binomial distribution for intravenous ketamine. We will use logistic regression to investigate the interaction between baseline pain, measured using the FPS-R, considered as a continuous variable, and treatment effect. All inferential analyses will be undertaken using a Bayesian framework with significance declared based on posterior probabilities. We will use $95 \%$ high-density posterior credible interval estimates to report treatment effect estimates. Due to the likelihood principal, no adjustments will be made for multiplicity and type I error will be controlled through prior specification. Descriptive statistics will be reported as frequencies and percentages for discrete variables and means, medians, SD and IQRs for continuous variables. A statistical analysis plan is being published separately and includes comprehensive details of prior specification. The primary analysis will determine if the optimal intranasal Ketodex combination is non-inferior to intravenous ketamine. All other analyses will test for superiority of intranasal Ketodex. The primary analysis of the primary outcome will involve logistic regression to model the dose response relationship for the combinations of intranasal Ketodex. The optimal dose combination will 


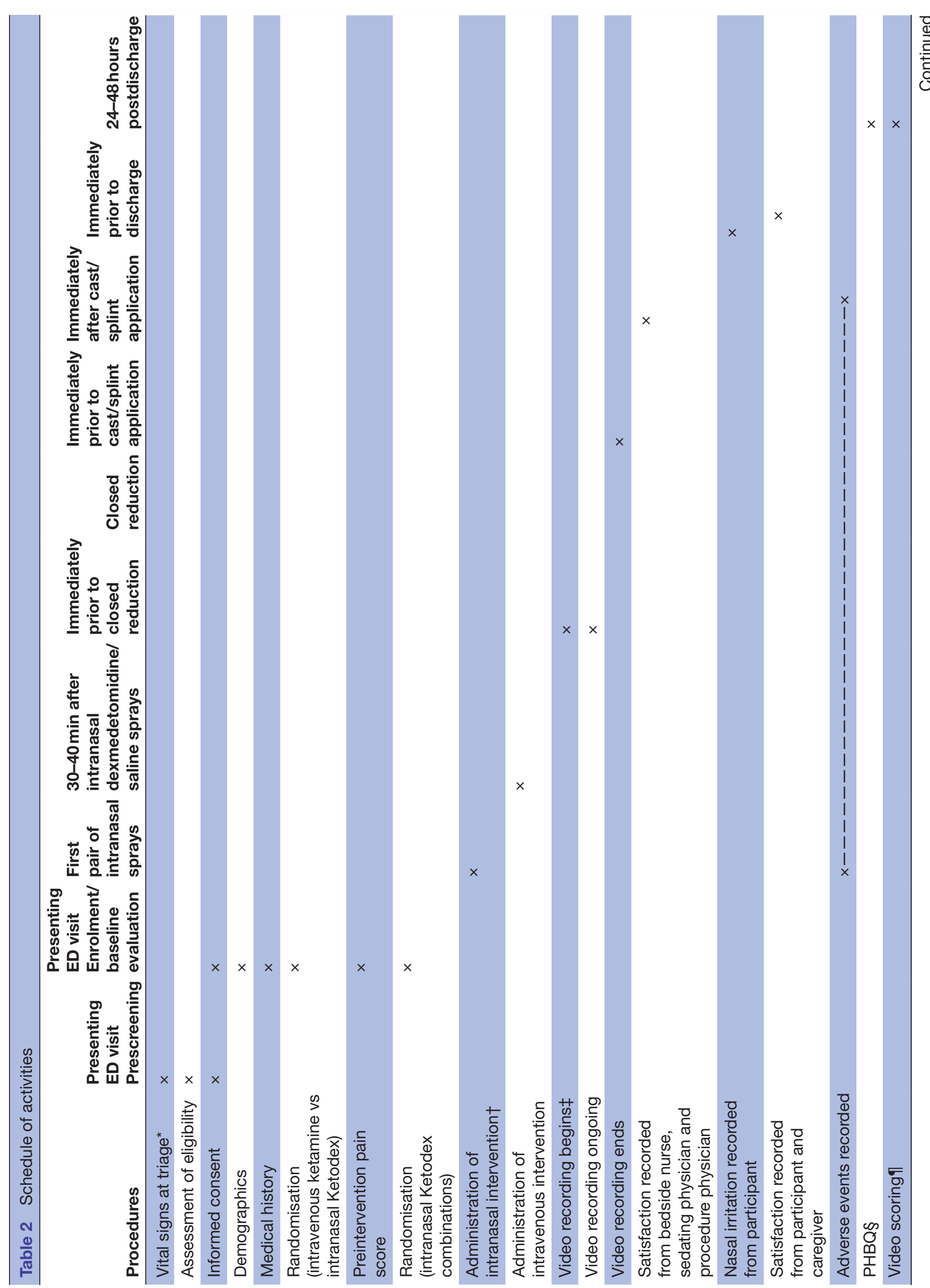






be the dose with the maximum posterior expected probability of adequate sedation. We will then consider whether this optimal dose is non-inferior to intravenous ketamine by computing the posterior probability of non-inferiority. If the probability of non-inferiority is above 0.97 , then we will declare that intranasal Ketodex is non-inferior to intravenous ketamine. This gives a power of 0.92 to detect that the probability of adequate sedation is equal to 0.9 and a type I error rate of $4.3 \%$. Secondary outcomes will be assessed using appropriate dose response models for intranasal Ketodex and posterior updating for intravenous ketamine. An additional analysis will investigate the interaction between baseline pain and the treatment effect.

\section{Interim analyses}

We will undertake seven interim analyses, at increments of 50 enrolled participants. Safety outcomes will be reviewed by the DSMB at each interim analysis. The decision to stop the trial for safety reasons will at the discretion of the DSMB. Due to the 'unbalanced' recruitment, the DSMB will not be blinded to treatment assignment. We will not undertake comparative effectiveness analyses at the interim analyses and will not stop for efficacy or futility.

\section{Missing data}

If the percentage of missing data is $\leq 5 \%$, we will undertake a per protocol analysis. If the percentage of missing data is $>5 \%$, we will use a full Bayesian analysis to jointly model the missing data model and the outcome model.

\section{Patient and public involvement}

The study team's patient engagement partner ( $\mathrm{SH}$ ) led a group of four patient partners who informed the trial's eligibility criteria, burden of interventions and outcomes. From their experiences as caregivers of children who visited a healthcare setting, they reviewed and provided feedback on the content of the recruitment pitch, letters of information, consent and assent. Results will not be directly disseminated to participants but will be provided on request or through access to the trial's website (www. kidscantrials.ca/ipctnetwork).

\section{Data management}

Data management services will be provided by the Women and Children's Health Research Institute (WCHRI) DCC. Study data will be entered and managed using REDCap tools hosted and supported by WCHRI. WCHRI's REDCap installation is a electronic, web-based data capture system validated in accordance with section C.05.012 of the Health Canada Part C, Division 5 of the Food and Drug Regulations, 'Drugs for Clinical Trials Involving Human Subjects'.

Data will be entered directly into the study database using a Wi-Fi-enabled encrypted iPad. In the case of a technical failure, data will be collected on paper and then transcribed into REDCap by the research nurse or site coordinator. 


\begin{tabular}{|c|l|}
\hline State & \multicolumn{1}{|c|}{ Behavior } \\
\hline $\mathbf{0}$ & $\begin{array}{l}\text { Sedation associated with abnormal physiologic parameters that require acute intervention (ie, oxygen saturation } \\
<90 \%, \text { blood pressure is } 30 \% \text { lower than baseline, bradycardia receiving therapy). }\end{array}$ \\
\hline $\mathbf{1}$ & $\begin{array}{l}\text { Deeply asleep with normal vital signs, but requiring airway intervention and/or assistance (eg, central or } \\
\text { obstructive apnea, etc). }\end{array}$ \\
\hline $\mathbf{2}$ & $\begin{array}{l}\text { Quiet (asleep or awake), not moving during procedure, and no frown (or brow furrow) indicating pain or anxiety. } \\
\text { No verbalization of any complaint. }\end{array}$ \\
\hline $\mathbf{3}$ & $\begin{array}{l}\text { Expression of pain or anxiety on face (may verbalize discomfort), but not moving or impeding completion of the } \\
\text { procedure. May require help positioning (as with a lumbar puncture) but does not require restraint to stop } \\
\text { movement during the procedure. }\end{array}$ \\
\hline $\mathbf{4}$ & $\begin{array}{l}\text { Moving during the procedure (awake or sedated) that requires gentle immobilization for positioning. May verbalize } \\
\text { some discomfort or stress, but there is no crying or shouting that expresses stress or objection. }\end{array}$ \\
\hline $\mathbf{5}$ & $\begin{array}{l}\text { Patient is moving (purposefully or no purposefully) in a manner that impedes the proceduralist and requires } \\
\text { forceful immobilization. This includes crying or shouting during the procedure, but vocalization is not required. } \\
\text { Score is based on movement. }\end{array}$ \\
\hline
\end{tabular}

From: Cravero JP, Askins N, Sriswasdi P, et al. Validation of the Paediatric Sedation State Scale. Paediatrics. 2017; 139(5):e20162897

Figure 2 Paediatric Sedation State Scale.

The study participant's contact information will be securely stored at each clinical site for internal use during the study. At the end of the study, all records will continue to be kept in a secure location for as long a period as dictated by the reviewing REB, institutional policies or sponsor requirements. For Health Canada-regulated trials, this is 25 years. Individual participants and their research data will be identified by a unique study identification number.

\section{Monitoring}

Monitoring for quality and regulatory compliance will be performed by the University of Alberta's Quality Management in Clinical Research (QMCR) office. QMCR is an independent unit housed within the university's central administration that provides arms-length review of all University of Alberta sponsored trials, at least three times per year. Details of clinical site monitoring will be documented in a clinical monitoring plan. This trial will also be monitored federally by Health Canada (approval number HC6-24-c230863) and an institutional level by site-specific clinical research oversight bodies, as per local requirements.

Safety oversight will be under the direction of the DSMB, which will function independently of the investigators. This committee will be chaired by Dr Garth Meckler and is composed of five individuals with expertise in trial methodology, epidemiology, biostatistics and paediatric emergency medicine. The DSMB will meet at least semiannually to assess safety and efficacy data and will operate under the rules of an approved charter/ terms of reference. Interim analyses will be undertaken at intervals of 50 enrolled participants. The DSMB will, in collaboration with the trial steering committee, establish safety stopping rules prior to trial initiation. The DSMB will be provided with a masked comparison between treatment groups with respect to the safety endpoints at the intervals of their choosing. At the DSMB's request, they can receive posterior credible intervals or predictive probabilities. They can further request unmasking. The decision to stop the trial for safety reasons will be left to the discretion of the DSMB.

\section{LIMITATIONS}

The most important limitation of our work is our inability to procure a higher concentration of ketamine for intranasal sprays. This will result in a large number of sprays for heavier participants. Although these individuals may be more compliant with a greater number of sprays, this may impact the external generalisability of the work and translation into clinical practice. Some countries (Australia and the USA) have access to higher concentrations of intranasal ketamine $(100 \mathrm{mg} / \mathrm{mL})$ for clinical use, which may increase the clinical uptake of our findings.

\section{ETHICS AND DISSEMINATION}

Ethics approval was obtained from Clinical Trials Ontario (London Health Sciences Centre and McMaster Research Ethics Board \#1987). The other participating sites have yet to receive ethics approval from their institutions. All protocol amendments will be submitted for approval to Health Canada before being communicated to each site. All protocol amendments will be added to the clinicaltrials.gov registration and implemented only after Health Canada and REB approval. All study participants, or their caregivers, will be notified if any new findings become available, which may be in the best medical interest of the study participant or may impact their willingness to continue participation in the study.

While long-term risks are not expected, immediate risks may occur. In terms of safety of intranasal dexmedetomidine, Kim $e t a l^{35}$ synthesised the evidence for preoperative intranasal dexmedetomidine in 11 trials of 1097 children and reported no occurrences of nausea, vomiting, 
hyperglycaemia, delirium or serious adverse effects. There have been no reports of mucosal irritation, ulceration or bleeding with intranasal administration. ${ }^{17}{ }^{36}$ All AEs will be classified according to MedDRA - a multilingual standardised international medical terminology dictionary used for 'regulatory communication and evaluation of data pertaining to medicinal products for human use. ${ }^{37}$ Serious adverse events (SAEs) will be defined based on the Quebec guidelines ${ }^{26}$ (see online supplemental file 3). Expected and unexpected SAEs will be reported to the DSMB and Health Canada, respectively.

The study team plans to publish results in a highimpact, peer-reviewed journal and present the findings at local research days, network meetings and societal conferences. The statistical analysis plan and design paper will be published separately. Additional dissemination strategies will be developed in conjunction with our patient partners and research team. The statistical code and dataset can be made available on request.

\section{Author affiliations}

${ }^{1}$ Departments of Paediatrics and Epidemiology \& Biostatistics, Schulich School of Medicine and Dentistry, London, Ontario, Canada

${ }^{2}$ Children's Health Research Institute, London Health Sciences Centre, London, Ontario, Canada

${ }^{3}$ Max Rady College of Medicine, Pediatrics and Child Health, Rady Faculty of Health Sciences, University of Manitoba, Winnipeg, Manitoba, Canada

${ }^{4}$ Department of Paediatrics, Children's Hospital Research Institute of Manitoba (CHRIM), Winnipeg, Manitoba, Canada

${ }^{5}$ Department of Paediatrics, Hospital for Sick Children, Toronto, Ontario, Canada

${ }^{6}$ Department of Paediatrics, University of Toronto, Toronto, Ontario, Canada

${ }^{7}$ Department of Statistical Science, University College London, London, UK

${ }^{8}$ Women and Children's Health Research Institute (WCHRI), University of Alberta,

Edmonton, Alberta, Canada

${ }^{9}$ Department of Paediatrics, Children's Hospital of Winnipeg, Winnipeg, Manitoba, Canada

${ }^{10}$ Department of Paediatrics, University of Ottawa, Children's Hospital of Eastern Ontario, Ottawa, Ontario, Canada

${ }^{11}$ Department of Paediatrics, McMaster University, McMaster Children's Hospital, Hamilton, Ontario, Canada

${ }^{12}$ Department of Paediatrics, University of British Columbia, BC Children's Hospital, Vancouver, British Columbia, Canada

${ }^{13}$ Child Health Evaluative Sciences, The Hospital for Sick Children, Toronto, Ontario, Canada

${ }^{14}$ Department of Pediatrics, Faculty of Medicine \& Dentistry, University of Alberta, Edmonton, Alberta, Canada

\section{Twitter Samina Ali @drsaminaali}

Acknowledgements The authors would like to acknowledge the IPCT SPOR administrative staff and our patient partners, who provided valuable support and input on the study design and documents. The following are the members of the KidsCAN PERC Innovative Pediatric Clinical Trials Ketodex Study Team: Dr Eleanor Pullenayegum, David Rios, Karly Stillwell, Shana Rimmer, Rick Watts, Tannis Erickson, Chelsea Bowkett, Carolyn Shimmin, Brendon Foot, Chelsea Bowkett, Candace McGahern, Redjana Carciurmaruj, Patricia Candelaria, and Jeannine Schellenberg.

Contributors NP is the principal investigator. He developed and revised the protocol, drafted the manuscript, and will oversee study operations. KC is a research coordinator. He contributed to study design and drafting of the manuscript. He will be responsible for all study operations. DB, SS, MB, AK, QD, VS and SA are site leads at their respective institutions. They contributed to study design and drafting of the protocol and manuscript. They will be responsible for study operations at their sites. M0 and PP contributed to the adaptive study methodology and drafting of the manuscript. AH and MY created the statistical analysis plan and contributed to drafting of the manuscript. TK is the nominated principal investigator for the IPCT-SPOR network. He reviewed and revised the manuscript. SH is a patient engagement partner. She led a group of other patient partners who provided input into patient-oriented outcomes, intervention administration and letters of information, consent and assent. All authors have approved the final version of the manuscript. None of the authors have financial or other conflicts of interests as they pertain to this study and its involved recruitment sites.

Funding This work is supported by an Innovative Clinical Trials Multi-year Grant from the Canadian Institutes of Health Research (funding reference number MYG-151207, 2017-2020), as part of the Strategy for Patient-Oriented Research and the Children's Hospital Research Institute of Manitoba (Winnipeg, Manitoba), the Centre Hospitalier Universitaire Sainte-Justine (Montreal, Quebec), the Department of Pediatrics, University of Western Ontario (London, Ontario), the Alberta Children's Hospital Research Institute (Calgary, Alberta), the Women and Children's Health Research Institute (Edmonton, Alberta), the Children's Hospital of Eastern Ontario Research Institute Inc. (Ottawa, Ontario) and the Hospital for Sick Children Research Institute (Toronto, Ontario). This study is sponsored by The Governors of the University of Alberta (Suite 400, 8215-112 Street, Edmonton, Alberta, Canada T6G 2C8). Neither the study sponsor nor funders have any role in the collection, management, analysis or interpretation of data; writing of the report; or the decision to submit the report for publication. Additional support was received from the Physicians Services Incorporated Foundation, Academic Medical Organization of Southwestern Ontario, Ontario Ministry of Economic Development, Job Creation and Trade, and the Children's Health Foundation of the Children's Hospital, London Health Sciences Foundation.

Competing interests None declared.

Patient consent for publication Not required.

Provenance and peer review Not commissioned; externally peer reviewed.

Supplemental material This content has been supplied by the author(s). It has not been vetted by BMJ Publishing Group Limited (BMJ) and may not have been peer-reviewed. Any opinions or recommendations discussed are solely those of the author(s) and are not endorsed by BMJ. BMJ disclaims all liability and responsibility arising from any reliance placed on the content. Where the content includes any translated material, BMJ does not warrant the accuracy and reliability of the translations (including but not limited to local regulations, clinical guidelines, terminology, drug names and drug dosages), and is not responsible for any error and/or omissions arising from translation and adaptation or otherwise.

Open access This is an open access article distributed in accordance with the Creative Commons Attribution Non Commercial (CC BY-NC 4.0) license, which permits others to distribute, remix, adapt, build upon this work non-commercially, and license their derivative works on different terms, provided the original work is properly cited, appropriate credit is given, any changes made indicated, and the use is non-commercial. See: http://creativecommons.org/licenses/by-nc/4.0/.

ORCID iDs

Naveen Poonai http://orcid.org/0000-0003-1540-0413

Samina Ali http://orcid.org/0000-0002-0595-364X

\section{REFERENCES}

1 Chamberlain JM, Patel KM, Pollack MM, et al. Recalibration of the pediatric risk of admission score using a multi-institutional sample. Ann Emerg Med 2004;43:461-8.

2 Hayden JC, Breatnach C, Doherty DR, et al. Efficacy of $\alpha 2$-agonists for sedation in pediatric critical care: a systematic review. Pediatr Crit Care Med 2016;17:e66-75.

3 Rennie L, Court-Brown CM, Mok JYQ, et al. The epidemiology of fractures in children. Injury 2007;38:913-22.

4 Cheng JC, Shen WY. Limb fracture pattern in different pediatric age groups: a study of 3,350 children. J Orthop Trauma 1993;7:15-22.

5 Jones K, Weiner DS. The management of forearm fractures in children: a plea for conservatism. J Pediatr Orthop 1999;19:811.

6 Havidich JE, Cravero JP. The current status of procedural sedation for pediatric patients in out-of-operating room locations. Curr Opin Anaesthesiol 2012;25:453-60.

7 Couloures KG, Beach M, Cravero JP, et al. Impact of provider specialty on pediatric procedural sedation complication rates. Pediatrics 2011;127:e1154-60.

8 Cummings EA, Reid GJ, Finley GA, et al. Prevalence and source of pain in pediatric inpatients. Pain 1996;68:25-31.

9 Dychter SS, Gold DA, Carson D, et al. Intravenous therapy: a review of complications and economic considerations of peripheral access. J Infus Nurs 2012;35:84-91. 
10 Graudins A, Meek R, Egerton-Warburton D, et al. The PICHFORK (pain in children fentanyl or ketamine) trial: a randomized controlled trial comparing intranasal ketamine and fentanyl for the relief of moderate to severe pain in children with limb injuries. Ann Emerg Med 2015;65:248-54

11 Schofield S, Schutz J, Babl FE, et al. Procedural sedation and analgesia for reduction of distal forearm fractures in the paediatric emergency department: a clinical survey. Emerg Med Australas 2013;25:241-7.

12 Nielsen BN, Friis SM, Rømsing J, et al. Intranasal sufentanil/ketamine analgesia in children. Paediatr Anaesth 2014;24:170-80.

13 Bilgen S, Köner Özge, Karacay S, et al. Effect of ketamine versus alfentanil following midazolam in preventing emergence agitation in children after sevoflurane anaesthesia: a prospective randomized clinical trial. J Int Med Res 2014:42:1262-71.

14 Ibrahim M, A prospective IM. A prospective, randomized, double blinded comparison of intranasal dexmedetomodine vs intranasa ketamine in combination with intravenous midazolam for procedural sedation in school aged children undergoing MRI. Anesth Essays Res 2014;8:179-86.

15 Kim HJ, Shin WJ, Park S, Ahn HS, et al. The sedative effects of the intranasal administration of dexmedetomidine in children undergoing surgeries compared to other sedation methods: a systematic review and meta-analysis. J Clin Anesth 2017;38:33-9.

16 Ter Bruggen FFJA, Eralp I, Jansen CK, et al. Efficacy of dexmedetomidine as a sole sedative agent in small diagnostic and therapeutic procedures: a systematic review. Pain Pract 2017;17:829-40.

17 Spohn J, Hendrikx S, Doyon-Trottier E, et al. LO62: intranasal dexmedetomidine for procedural distress in children: a systematic review and meta-analysis. CJEM 2019;21:S30.

18 Qiao H, Xie Z, Jia J. Pediatric premedication: a double-blind randomized trial of dexmedetomidine or ketamine alone versus a combination of dexmedetomidine and ketamine. BMC Anesthesiol 2017;17:158-65.

19 Bhat R, Santhosh MCB, Annigeri VM, Rao RP, et al. Comparison of intranasal dexmedetomidine and dexmedetomidine-ketamine for premedication in pediatrics patients: a randomized double-blind study. Anesth Essays Res 2016;10:349-55.

20 Frey TM, Florin TA, Caruso M, et al. Effect of intranasal ketamine vs fentanyl on pain reduction for extremity injuries in children: the prime randomized clinical trial. JAMA Pediatr 2019;173:140-6.

21 Poonai N, Spohn J, Vandermeer B, et al. Intranasal dexmedetomidine for anxiety-provoking procedures in children: a systematic review and meta-analysis. Pediatrics 2020;145:e20191623.

22 Calvert M, Kyte D, Mercieca-Bebber R, et al. Guidelines for inclusion of patient-reported outcomes in clinical trial protocols: the SPIRITPRO extension. JAMA 2018;319:483-94.
23 Lewis RJ, Viele K, Broglio K, et al. An adaptive, phase II, dose-finding clinical trial design to evaluate L-carnitine in the treatment of septic shock based on efficacy and predictive probability of subsequent phase III success. Crit Care Med 2013;41:1674-8.

24 Green SM, Roback MG, Kennedy RM, et al. Clinical practice guideline for emergency department ketamine dissociative sedation: 2011 update. Ann Emerg Med 2011;57:449-61.

25 Herd DW, Anderson BJ, Keene NA, et al. Investigating the pharmacodynamics of ketamine in children. Paediatr Anaesth 2008;18:36-42.

26 Bhatt M, Kennedy RM, Osmond MH, et al. Consensus-Based recommendations for standardizing terminology and reporting adverse events for emergency department procedural sedation and analgesia in children. Ann Emerg Med 2009;53:426-35.

27 Quibell R, Prommer EE, Mihalyo M, et al. Ketamine*. J Pain Symptom Manage 2011;41:640-9.

28 Strayer RJ, Nelson LS. Adverse events associated with ketamine for procedural sedation in adults. Am J Emerg Med 2008;26:985-1028.

29 Kankkunen P, Vehviläinen-Julkunen K, Pietilä AM, et al. Parents perceptions and use of analgesics at home after children's day surgery. Pediatric Anesthesia 2003;13:132-40.

30 Cravero JP, Askins N, Sriswasdi P, et al. Validation of the pediatric sedation state scale. Pediatrics 2017;139:e20162897.

31 Coté CJ, Wilson S. American academy of pediatrics, American Academy of pediatric dentistry. guidelines for monitoring and management of pediatric patients before, during, and after sedation for diagnostic and therapeutic procedures: update 2016. Pediatrics 2016;138:e1-31.

32 Kaplan RF. Sedation/Analgesia for diagnostic and therapeutic procedures in children outside of the operating room. ASA Refresher Courses in Anesthesiology 2006;34:77-83.

33 Hicks CL, von Baeyer CL, Spafford PA, et al. The faces pain scalerevised: toward a common metric in pediatric pain measurement. Pain 2001;93:173-83.

34 Joseph L, Belisle P. Bayesian sample size determination for normal means and differences between normal means. The Statistician 1997:46:209-66.

$35 \mathrm{Kim}$ HJ, Shin WJ, Park S, et al. The sedative effects of the intranasal administration of dexmedetomidine in children undergoing surgeries compared to other sedation methods: a systematic review and metaanalysis. J Clin Anesth 2017;38:33-9.

36 Liu Y, Yu Q, Sun M, et al. Median effective dose of intranasal dexmedetomidine sedation for transthoracic echocardiography examination in postcardiac surgery and normal children: an up-anddown sequential allocation trial. Eur J Anaesthesiol 2018;35:43-8.

37 International Council for Harmonisation of Technical Requirements for Pharmaceuticals for Human Use $(\mathrm{ICH})$. Medical dictionary for regulatory activities (MeDRA) 2019, 2019 